\title{
The Early Histological Effects of Intravesical Instillation of Platelet- Rich Plasma in Cystitis Models
}

\author{
M. İrfan Dönmez ${ }^{1}$, Kubilay İnci ${ }^{1, \dagger}$, Naciye Dilara Zeybek ${ }^{2}$, H. Serkan Doğan ${ }^{1}$, Ali Ergen ${ }^{1}$ \\ ${ }^{1}$ Department of Urology, Hacettepe University School of Medicine, Ankara, Turkey \\ ${ }^{2}$ Department of Histology and Embryology, Hacettepe University School of Medicine, Ankara, Turkey
}

Purpose: To evaluate the early histological effects of the intravesical instillation of platelet-rich plasma (PRP) in rabbit models of interstitial and hemorrhagic cystitis.

Methods: Thirty-six rabbits were classified into 6 groups: saline (S), S+PRP, hydrochloric acid ( $\mathrm{HCl}), \mathrm{HCl}+\mathrm{PRP}$, cyclophosphamide (CyP), and CyP+PRP. At 48 hours after induction, $P R P$ was prepared and intravesically administered to the S+PRP, $\mathrm{HCl}+\mathrm{PRP}$, and $\mathrm{CyP}+\mathrm{PRP}$ groups. Bladder sections were stained with toluidine blue for mast cell counting and with hematoxylin and eosin for histopathology and mitotic index determination. The proliferation index was determined by proliferating cell nuclear antigen (PCNA) immunolabeling. The nonparametric Mann-Whitney U-test was used for statistical analysis.

Results: No abnormalities were observed in the $S$ group, whereas increased interstitial edema and increased average mitotic and proliferation indices were observed in the $\mathrm{S}+\mathrm{PRP}$ group $(\mathrm{P}=0.023, \mathrm{P}=0.004$, and $\mathrm{P}=0.009$, respectively). Intense epithelial loss, hemorrhage, and leukocyte infiltration were detected in the $\mathrm{HCl}$ and $\mathrm{HCl}+\mathrm{PRP}$ groups, whereas a significantly increased average mitotic index was observed in the $\mathrm{HCl}+\mathrm{PRP}$ group $(\mathrm{P}=0.002)$. When compared with its CyP counterpart, a significant reduction in hemorrhage and an increase in leukocyte infiltration and mitotic index were observed in the $\mathrm{CyP}+\mathrm{PRP}$ group $(\mathrm{P}=0.006, \mathrm{P}=0.038$, and $\mathrm{P}=0.002$, respectively). In addition, $\mathrm{PCNA}$ staining revealed a significantly increased proliferation index in the $\mathrm{HCl}+\mathrm{PRP}$ and $\mathrm{CyP}+\mathrm{PRP}$ groups $(\mathrm{P}=0.032$ and $\mathrm{P}=0.015$, respectively).

Conclusions: The intravesical instillation of PRP increased the mitotic index in the saline and cyclophosphamide groups while decreasing macroscopic bleeding.

Keywords: Cystitis; Cystitis, Interstitial; Administration, Intravesical

- Fund Support: This study was funded by Hacettepe University Scientific Research Unit.

- Research Ethics: All applicable international, national, and/or institutional guidelines for the care and use of animals were followed. All experiments in this study were performed in accordance with the local guidelines for animal research from the National Institutes of Health and were approved by the Local Hospital Ethics Committee (B.30.2.HAC.0.05.06.00/15-2012/58).

- Conflict of Interest: No potential conflict of interest relevant to this article was reported.

\section{INTRODUCTION}

Despite extensive research, the etiology, diagnosis, and treatment of interstitial cystitis/bladder pain syndrome (IC/BPS) have not been clearly established. Theories about the etiology of
IC/BPS are inadequate; thus, diagnosis and treatment methods are equally unsatisfactory. As a result, the diagnosis can be delayed for as long as 5 years, with consequent disease progression and reduction in the benefits of existing treatments [1].

Platelet-rich plasma (PRP) is leukocyte-free autologous plas-
Corresponding author: M. İrfan Dönmez (D) http://orcid.org/0000-0002-2828-7942 Department of Urology, Hacettepe University School of Medicine, Sihhiye, Ankara 06100, Turkey

E-mail: m_irfan83@yahoo.com / Tel: +90-312-305 1969 / Fax: +90-312-311 2262 ${ }^{\dagger}$ Deceased.

Submitted: February 14, 2016 / Accepted after revision: May 2, 2016 
ma mixed with plasma proteins prepared from the patient's own blood. It contains factors, such as platelet-derived growth factor (PDGF), transforming growth factor (TGF), insulin-like growth factor (IGF), and epidermal growth factor (EGF), that initiate wound healing and tissue repair as well as plasma proteins, such as fibrin, fibronectin, and vitronectin, that contribute to hemostasis and adhesion. The factors in PRP stimulate and organize healing, cellular mitogenesis, chemotaxis, differentiation, and metabolism [2]. Within the last 2 decades, PRP has drawn remarkable interest from medical specialties including sports medicine, orthopedics, and maxillofacial surgery because of its wound-healing effects [3]. Despite all this evidence, the full biologic content of PRP is not yet clearly understood [4].

Because the etiology of IC is linked to urothelial defects, we hypothesized that the intravesical instillation of PRP would induce the regeneration of the bladder urothelium. The aim of this study was to show the early histological effects of PRP in cystitis in a New Zealand White rabbit model and to obtain data on the outcomes of intravesical instillation of PRP.

\section{MATERIALS AND METHODS}

Thirty-six male New Zealand white rabbits weighing between 2,800 and 3,200 g and aged between 6 and 12 months were randomly classified into 6 groups with 6 rabbits in each group: saline (S), S+PRP, hydrochloric acid ( $\mathrm{HCl}), \mathrm{HCl}+\mathrm{PRP}$, cyclophosphamide ( $\mathrm{CyP})$, and $\mathrm{CyP}+\mathrm{PRP}$. The saline group was used as a control, the $\mathrm{HCl}$ group to mimic interstitial cystitis, and cyclophosphamide to produce hemorrhagic cystitis. The animals were maintained on 12-hour light/dark cycle with free access to food and water.

The animals' bladders were emptied prior to instillation, and the catheters were removed after 4 minutes to ensure that they did not void the subsequently instilled material. No sedation was used before the intravesical instillation. The bladder capacity of the animals was approximately $50 \mathrm{~mL}$, and one-sixth of the total bladder capacity volume was used for intravesical instillation [5]. The S and S+PRP groups were intravesically administered $8 \mathrm{~mL}$ of physiologic serum via a sterile technique using a 6-Fr urethral catheter. The $\mathrm{HCl}$ and $\mathrm{HCl}+\mathrm{PRP}$ groups were administered $1 \mathrm{~mL}$ of $0.4 \mathrm{~N} \mathrm{HCl}$ using the same sterile technique, and the $\mathrm{HCl}$ was removed after 1 minute. The $\mathrm{CyP}$ and $\mathrm{CyP}+\mathrm{PRP}$ groups received an intraperitoneal injection of $300 \mathrm{mg} / \mathrm{kg}$ of CyP.

PRP was prepared as described by Nagae et al. [6]. Under sedation, the intracardiac route was used to withdraw blood because the filling rate for superficial veins would have been very slow and coagulation would have begun immediately during filling. A mean of $4.5 \mathrm{~mL}$ (3.5-6.0 mL) of blood was immediately injected into $4.5-\mathrm{mL}$ tubes containing 3.2\% citrate. Blood samples were obtained prior to the instillation and were immediately sent to the laboratory to prepare PRP. The samples were centrifuged at 1,500 rpm $(250 \times \mathrm{g})$ for 10 minutes to achieve separation of the supernatant and buffy coat. The remaining plasma was then centrifuged at 3,000 $\mathrm{rpm}(1,000 \times \mathrm{g})$ for another 10 minutes, and the thrombocytes were precipitated. The supernatant, which contained fewer platelets, was discarded, and a mean $1 \mathrm{~mL}(0.8-1.2 \mathrm{~mL})$ of PRP was obtained from the buffy coat. PRP was intravesically administered using a 6-Fr sterile self-lubricating catheter via the sterile technique.

A total of 48 hours after saline and $\mathrm{HCl}$ had been administered, PRP was instilled to the $\mathrm{S}+\mathrm{PRP}, \mathrm{HCl}+\mathrm{PRP}$, and $\mathrm{CyP}+\mathrm{PRP}$ groups via the sterile technique. The bladders of the animals in the $\mathrm{S}$ and $\mathrm{HCl}$ groups were surgically removed 96 hours after the instillation, whereas those of the animals in the CyP group were removed at the 72nd hour of administration. Animals in the remaining groups were sacrificed after 48 hours of PRP in-

Table 1. Working schedule

\begin{tabular}{lccccc}
\hline Group & Starting time & 24 Hours & 48 Hours & 72 Hours & 96 Hours \\
\hline S & S & - & - & - & Sacrified \\
S+PRP & S & - & PRP & - & Sacrified \\
$\mathrm{HCl}$ & $\mathrm{HCl}$ & - & - & - & Sacrified \\
$\mathrm{HCl}+\mathrm{PRP}$ & $\mathrm{HCl}$ & - & PRP & - & Sacrified \\
CyP & $\mathrm{CyP}$ & - & - & Sacrified & \\
Cyp+PRP & CyP & PRP & - & Sacrified & \\
\hline
\end{tabular}

S, saline; $\mathrm{PRP}$, platelet rich plasma; $\mathrm{HCl}$, hydrochloric acid; $\mathrm{CyP}$, cyclophosphamide. 
stillation. The working schedule is briefly explained in Table 1.

The surgical procedure was performed after the intraperitoneal application of $5 \mathrm{mg} / \mathrm{kg}$ of ketamine. The midumbilical line was incised $5 \mathrm{~cm}$ above the bladder. After dissecting the muscular layers and opening the peritoneum, the fatty tissue adjacent to the bladder was dissected and the bladder was removed just below the bladder neck. The animals were sacrificed using $\mathrm{CO}_{2}$.

Samples from the urinary bladder were fixed in 10\% neutral buffered formalin solution, dehydrated through graded alcohols, and subsequently embedded in paraffin blocks. Sections ( 5 $\mu \mathrm{m})$ were cut and stained with toluidine blue for mast cell counting and with hematoxylin and eosin for histopathology and mitotic index determination according to standard protocol. Stained tissue sections were examined and photographed using a light microscope (Leica DM6000B, Leica, Wetzlar, Germany) with a DC490 digital camera (Leica). The histologist was blinded to the groups. The severity of inflammation was determined in 10 fields of each section on the basis of 4 criteria: mucosal abrasion, hemorrhage, leukocyte infiltration, and edema [7].

The presence of mucosal abrasion and hemorrhage at $100 \times$ magnification was scored as 1 , and no change was scored as 0 .

Leukocyte infiltration (neutrophils and mononuclear cells) was evaluated in the mucosa. In each of the fields of view at $\times 400$ magnification, no extravascular leukocytes were scored as $0,>20$ leukocytes as $1,20-45$ leukocytes as 2 , and $>45$ leukocytes as 3 .

Edema was evaluated under $\times 200$ magnification. No edema was scored as 0 , minimal edema (no change in connective tissue thickness) as 1, moderate edema (connective tissue thickness increased by $<2$ fold) as 2 , and severe edema (connective tissue thickness increased by $>2$ fold) as 3 .

The total score of all fields of view for mucosal abrasion, hemorrhage, leukocyte infiltration, and edema was divided by the maximum possible score and multiplied by 100 . The average mitotic index was calculated as the average number of mitotic figures per 10 fields of urothelium with $\times 400$ magnification [8].

For immunolabeling of the urothelium with proliferating cell nuclear antigen (PCNA), paraffin sections of 5- $\mu \mathrm{m}$ thickness were incubated at $60^{\circ} \mathrm{C}$ overnight and rinsed in xylene for 15 minutes. After rehydrating through decreasing concentrations of ethanol, the sections were washed with $0.01 \mathrm{M}$ phosphatebuffered saline (PBS) at $\mathrm{pH} 7.4$ for 5 minutes. Heat-mediated antigen retrieval was performed in a microwave for 15 minutes at $600 \mathrm{~W}$ in $10 \mathrm{mM}$ citrate buffer $(\mathrm{pH}, 6.0)$. Sections were cooled and washed with PBS 3 times for 5 minutes. Endogenous peroxidase activity was blocked by incubation in absolute methanol containing $3 \% \mathrm{H}_{2} \mathrm{O}_{2}$ for 5 minutes at $40^{\circ} \mathrm{C}$. After rinsing several times in PBS, nonspecific binding sites were blocked with 10\% normal goat serum (Cat No. 50-062Z, Invitrogen, Camarillo, CA, USA) for 10 minutes. The sections were then incubated with anti-mouse PCNA primary antibody (Cat No. 13-3900, Invitrogen) for 1 hour at room temperature. After washing with PBS 3 times for 5 minutes, sections were incubated with a 1:200 dilution of goat anti-mouse IgG (Cat No. 31160, Pierce Inc., Rockford, IL, USA) in PBS containing 0.2\% BSA (Cat No. A-7034, Sigma, Saint Louis, MO, USA) and 1\% normal rabbit serum for 30 minutes at room temperature. After washing in PBS, peroxidase activity was revealed through incubation for 5 minutes with DAB (Cat No. 00-2014, Zymed, San Francisco, CA, USA) and counterstaining with Mayer's hematoxylin. Following washing with tap water, sections were dehydrated through graded alcohols and cleared in xylene prior to mounting with Entellan (Cat No. 100869, Merck, Darmstadt, Germany). Negative controls were prepared by omitting the incubation with the primary antibody.

Three different epithelial areas of each slide were evaluated under a microscope at $\times 200$ original magnification. The quantification of PCNA-positive cells was accomplished by counting the number of PCNA-positive nuclei observed in the microscopic fields while counting 100 cells to determine the labeling index. The proliferation index was calculated as the number of PCNA-labeled mitotic cells divided by the total number of cells counted (labeled cells/100 cells) [8].

The values of the variables of interest to be compared between groups were not normally distributed; therefore, we used the Mann-Whitney U-test, which is appropriate for nonparametric data, to evaluate the significance of differences in these variables between groups. A P-value $<0.05$ was considered statistically significant. All numeric values were tested for normal distrubution. Data are shown as mean \pm standard deviation. Statistical analysis was performed using SPSS ver. 13 (SPSS, Inc.; Chicago, IL, USA).

\section{RESULTS}

After the extraction of the bladders, the $\mathrm{S}$ and $\mathrm{S}+\mathrm{PRP}$ groups were macroscopically identical. However, hemorrhage and he- 

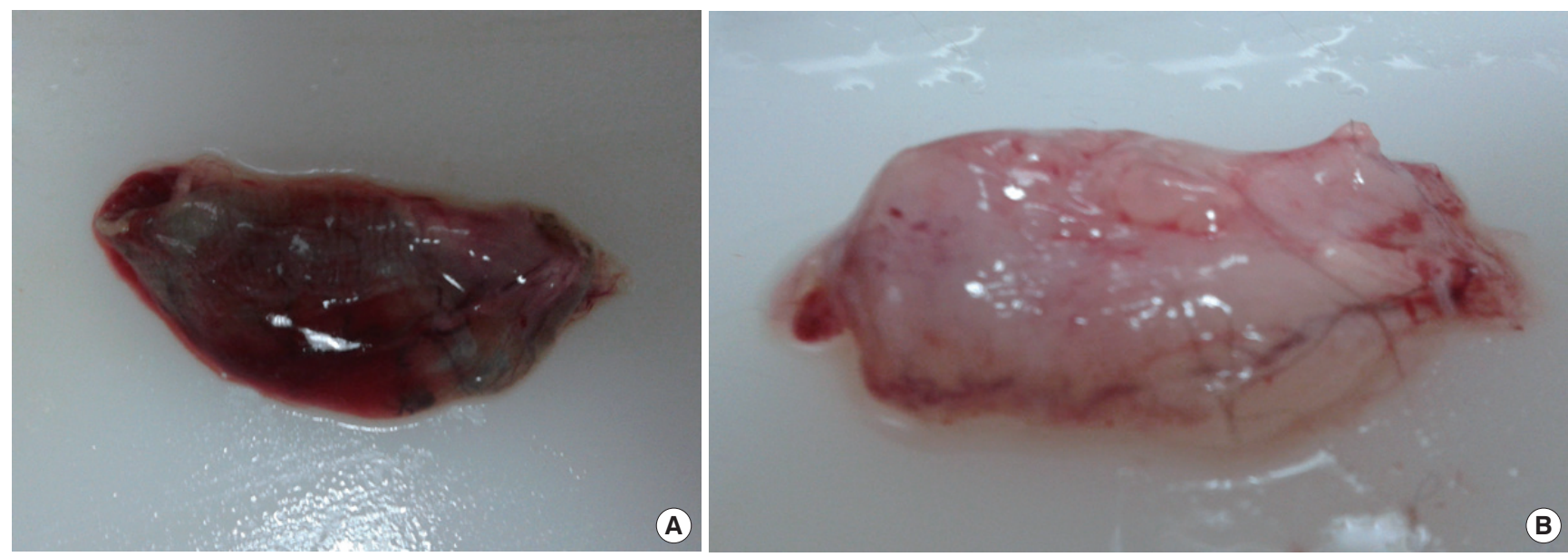

Fig. 1. Macroscopic view of a urinary bladder from the $\mathrm{CyP}(\mathrm{A})$ and $\mathrm{CyP}+\mathrm{PRP}(\mathrm{B})$ groups. $\mathrm{CyP}$, cyclophosphamide; PRP, platelet-rich plasma.

Table 2. Histologic changes between groups

\begin{tabular}{|c|c|c|c|c|c|c|c|c|c|}
\hline Variable & $\begin{array}{c}S \\
(n=6)\end{array}$ & $\begin{array}{c}S+P R P \\
(n=6)\end{array}$ & P-value ${ }^{a)}$ & $\begin{array}{c}\mathrm{HCl} \\
(\mathrm{n}=6)\end{array}$ & $\begin{array}{c}\mathrm{HCl}+\mathrm{PRP} \\
(\mathrm{n}=6)\end{array}$ & P-value ${ }^{\text {b) }}$ & $\begin{array}{c}\text { CyP } \\
(n=6)\end{array}$ & $\begin{array}{c}\mathrm{CyP}+\mathrm{PRP} \\
(\mathrm{n}=6)\end{array}$ & P-value ${ }^{c)}$ \\
\hline Mucosal abrasion & $10.00 \pm 10.95$ & $10.00 \pm 10.95$ & 1.000 & $78.33 \pm 19.40$ & $75.00 \pm 21.67$ & 0.871 & $68.33 \pm 17.22$ & $53.33 \pm 36.14$ & 0.572 \\
\hline Hemorrhage & $13.33 \pm 10.32$ & $13.33 \pm 12.11$ & 1.000 & $91.67 \pm 13.29$ & $81.67 \pm 22.28$ & 0.389 & $83.33 \pm 27.32$ & $15.00 \pm 16.43$ & $0.006^{*}$ \\
\hline $\begin{array}{l}\text { Leucocyte } \\
\text { infiltration }\end{array}$ & $8.88 \pm 8.60$ & $13.88 \pm 15.40$ & 0.623 & $80.00 \pm 18.37$ & $92.22 \pm 14.24$ & 0.411 & $40.00 \pm 5.16$ & $48.33 \pm 7.52$ & $0.038^{*}$ \\
\hline Edema & $9.44 \pm 12.00$ & $41.66 \pm 26.47$ & $0.023^{*}$ & $87.77 \pm 20.51$ & $95.55 \pm 5.01$ & 0.675 & $83.33 \pm 18.25$ & $88.33 \pm 16.83$ & 0.789 \\
\hline Mitotic index & $0.86 \pm 43(0-3)$ & $1.50 \pm 0.26(1-5)$ & $0.004^{*}$ & $0.10 \pm 0(0-1)$ & $1.66 \pm 0.17(0-3)$ & $0.002^{*}$ & $0.10 \pm 0(0-1)$ & $1.51 \pm 0.53(0-3)$ & $0.002^{*}$ \\
\hline Proliferation index & $42.7 \pm 9.93$ & $62.90 \pm 10.50$ & $0.009^{*}$ & $48.65 \pm 4.12$ & $66.34 \pm 10.65$ & $0.032^{*}$ & $42.7 \pm 10.33$ & $59.7 \pm 8.64$ & $0.015^{*}$ \\
\hline
\end{tabular}

Values are presented as mean \pm standard deviation $(\mathrm{SD})$ or mean $\pm \mathrm{SD}$ (range).

The severity of inflammation was determined according to five criteria, including mucosal abrasion, haemorrhage, leukocyte infiltration, mitotic index and oedema. Proliferation index using PCNA was calculated as the number of labeled cells divided by total cells counted (labeled cells/100 cell). Mitotic index was analysed by counting the mitotic figures per 10 high-power fields.

$\mathrm{S}$, saline; $\mathrm{PRP}$, platelet rich plasma; $\mathrm{HCl}$, hydrochloric acid; $\mathrm{CyP}$, cyclophosphamide.

${ }^{a)} \mathrm{S}$ vs. $\mathrm{S}+\mathrm{PRP} ;{ }^{\text {b) }} \mathrm{HCl}$ vs. $\mathrm{HCl}+\mathrm{PRP} ;{ }^{\mathrm{c})} \mathrm{CyP}$ vs. $\mathrm{CyP}+\mathrm{PRP} .{ }^{*} \mathrm{P}<0.05$, statistical significant difference.

maturia were evident by visual inspection in the $\mathrm{HCl}$ and $\mathrm{CyP}$ groups. Interestingly, there was no macroscopic hemorrhage or hematuria in the $\mathrm{HCl}+\mathrm{PRP}$ or $\mathrm{CyP}+\mathrm{PRP}$ groups (Fig. 1). Furthermore, the weights of the bladders ranged between 6 and $10 \mathrm{~g}$.

A normal morphology of the mucosa with an intact urothelium was observed in the $\mathrm{S}$ and $\mathrm{S}+\mathrm{PRP}$ groups (Fig. 2A, B). The $\mathrm{S}+\mathrm{PRP}$ group demonstrated significantly higher edema compared with the $S$ group $(\mathrm{P}=0.023)$ (Table 2, Fig. 2).

The instillation of $\mathrm{HCl}$ or $\mathrm{CyP}$ resulted in the loss of urothelial cells. The $\mathrm{HCl}$ model caused more severe mucosal abrasion with locally denuded and ulcerated areas than the CyP model and $\mathrm{S}$ group. The congestion of blood vessels was observed in the $\mathrm{HCl}$ and $\mathrm{CyP}$-induced cystitis models (Figs. 2, 3). The instil- lation of $\mathrm{HCl}$ and $\mathrm{CyP}$ resulted in hemorrhage, leukocyte infiltration, and edema within the bladder wall, which were significant changes when compared with those in the $S$ group $(\mathrm{P}=0.003, \mathrm{P}=0.004$, and $\mathrm{P}=0.004$ for $\mathrm{HCl}$ and $\mathrm{P}=0.003, \mathrm{P}=$ 0.004 , and $\mathrm{P}=0.003$ for $\mathrm{CyP}$, respectively).

$\mathrm{PRP}$ administration to the $\mathrm{HCl}$ and $\mathrm{CyP}$ models decreased the severity of mucosal abrasion and hemorrhage and caused an increase in the severity of leukocyte infiltration and edema. The decrease in the severity of mucosal abrasion and hemorrhage and increase in leukocyte infiltration and edema in the $\mathrm{HCl}+\mathrm{PRP}$ group were not statistically significant compared with those in the $\mathrm{HCl}$ models. The decrease in hemorrhage and increase in leukocyte infiltration were statistically significant in 


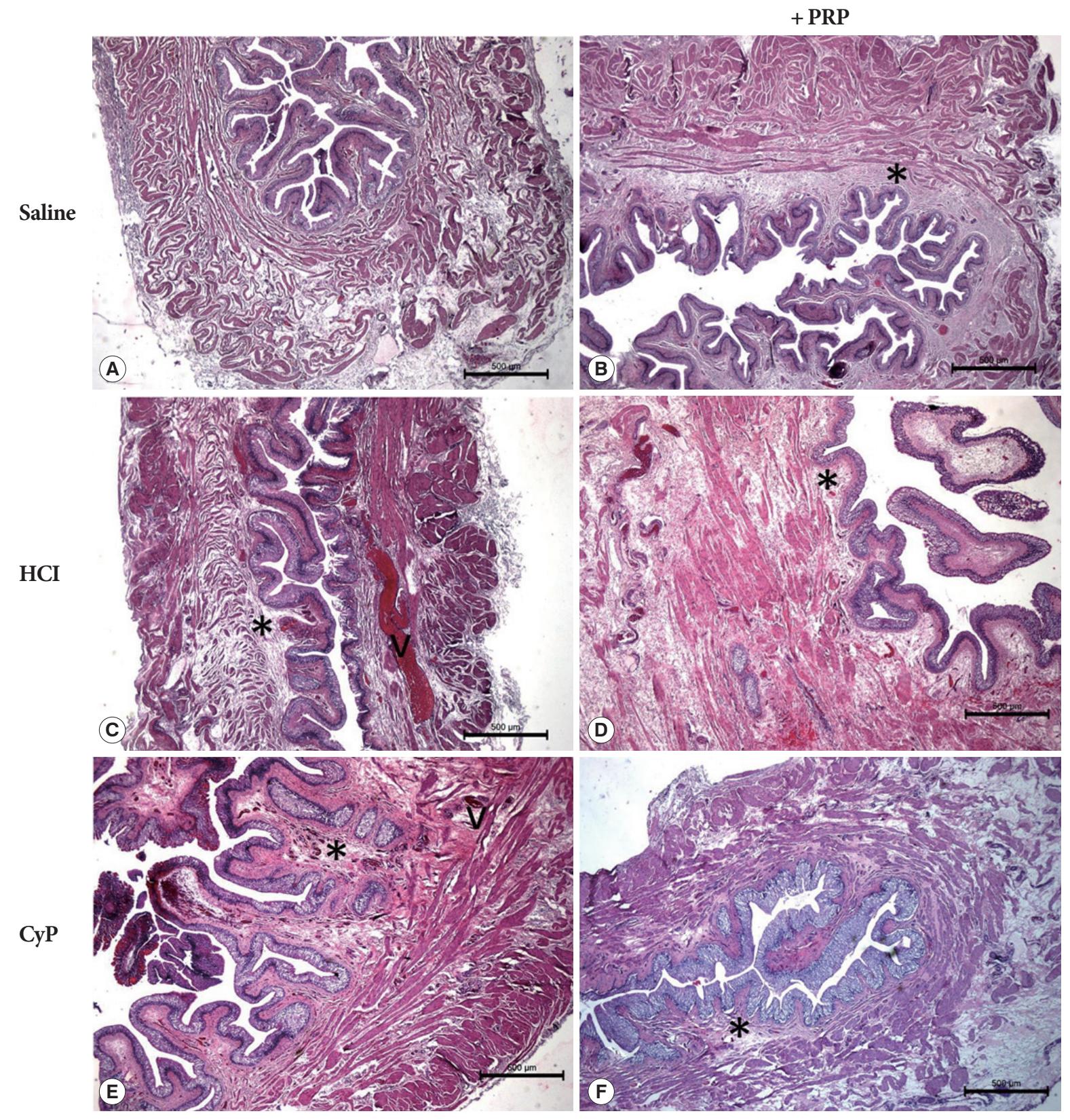

Fig. 2. Histopathologic changes in the cystitis models and the effects of PRP administration. Regular morphology of a urinary bladder with intact urothelium in the S (A) and S+PRP groups (B). Loss of urothelial cells, hemorrhage, and congested blood vessels (V) in the $\mathrm{HCl}(\mathrm{C})$ and $\mathrm{CyP}$ cystitis models (E). Edema $\left(^{*}\right)$ in the S+PRP group (B) and cystitis models (D, F). H\&E, original magnification, $\times 25$. S, saline; $\mathrm{PRP}$, platelet-rich plasma; $\mathrm{HCl}$, hydrochloric acid; $\mathrm{CyP}$, cyclophosphamide.

the $\mathrm{CyP}+\mathrm{PRP}$ group compared with those in the $\mathrm{CyP}$ model $(\mathrm{P}=0.006$ and $\mathrm{P}=0.038$, respectively).

PRP administration significantly increased the mitotic index in the urothelium in all groups $(\mathrm{P}=0.004, \mathrm{P}=0.002$, and $\mathrm{P}=0.002$ for $\mathrm{S}$ vs. $\mathrm{S}+\mathrm{PRP}, \mathrm{HCl}$ vs. $\mathrm{HCl}+\mathrm{PRP}$, and $\mathrm{CyP}$ vs. $\mathrm{CyP}+$ $\mathrm{PRP}$, respectively).
A few mast cells were observed in the urinary bladder of the animals in the experimental groups. There was no significant difference between the number of mast cells in the cystitis models and PRP-treated groups.

We assessed the proliferation index in the urothelium using PCNA immunoreactivity for each group. We found that PRP 


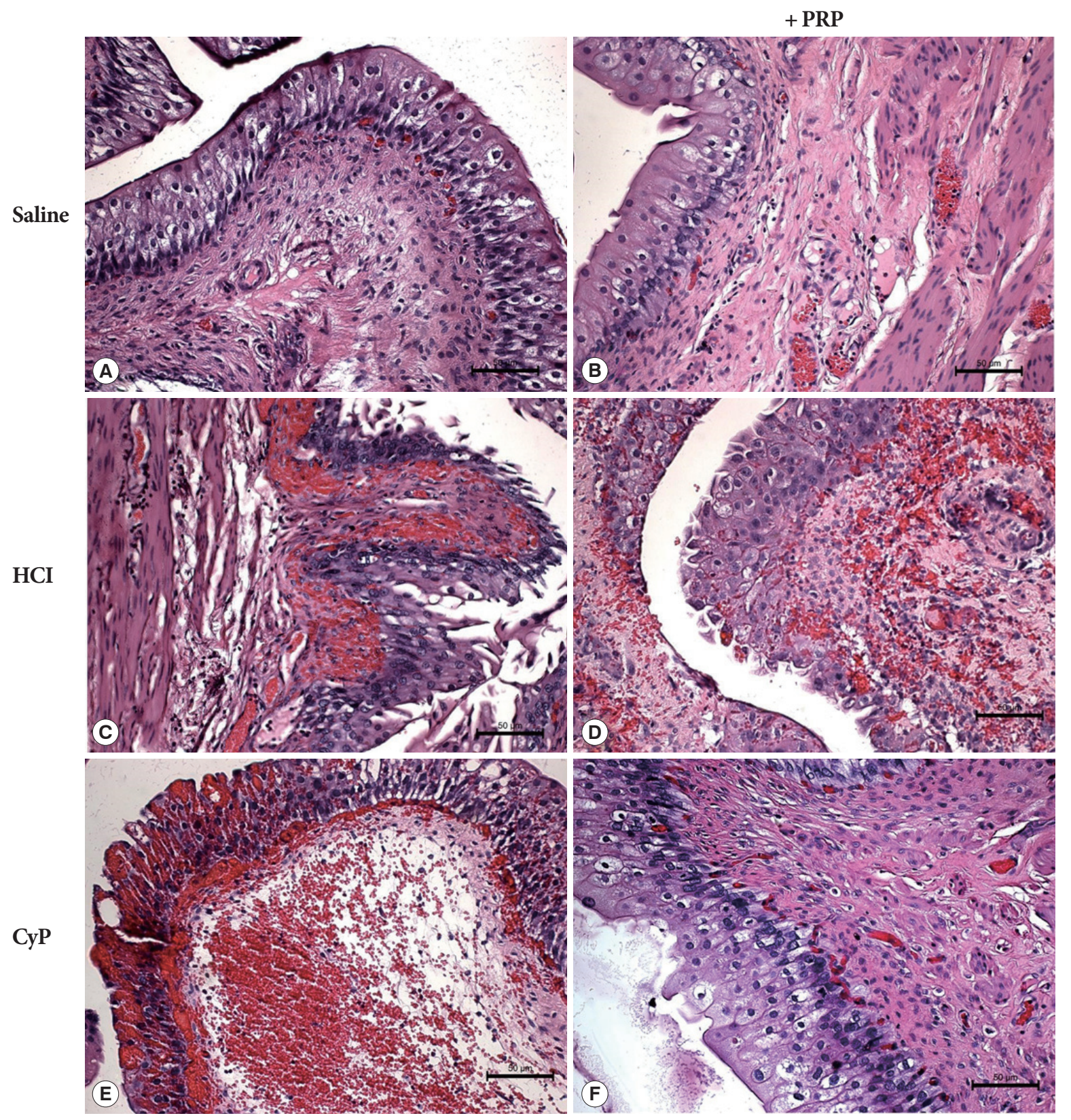

Fig. 3. Normal mucosa with intact urothelium and regular lamina propria in the $S$ (A) and S+PRP groups (B). Denuded epithelia and prominent hemorrhage in the $\mathrm{HCl}$ cystitis model (C). Hemorrhage and mucosal abrasion in the $\mathrm{HCl}+\mathrm{PRP}$ group (D). Degeneration of epithelial cells and prominent hemorrhage in the CyP cystitis model (E). Significantly decreased hemorrhage in the CyP+PRP group (F). H\&E, original magnification, $\times 200$. S, saline; $\mathrm{PRP}$, platelet-rich plasma; $\mathrm{HCl}$, hydrochloric acid; $\mathrm{CyP}$, cyclophosphamide.

administration significantly increased the PCNA-positive cell number in the urothelium compared with that in the cystitis models $(\mathrm{P}=0.009, \mathrm{P}=0.032$, and $\mathrm{P}=0.015$ for $\mathrm{S}$ vs. $\mathrm{S}+\mathrm{PRP}, \mathrm{HCl}$ vs. $\mathrm{HCl}+\mathrm{PRP}$, and CyP vs. CyP+PRP, respectively; Fig. 4).

\section{DISCUSSION}

In IC/BPS, treatment has to be symptom-related because its etiology is unclear. Several treatment options have been proposed; however, none have achieved notable success. The nature of the disease, which causes a chronic pain syndrome independent of 

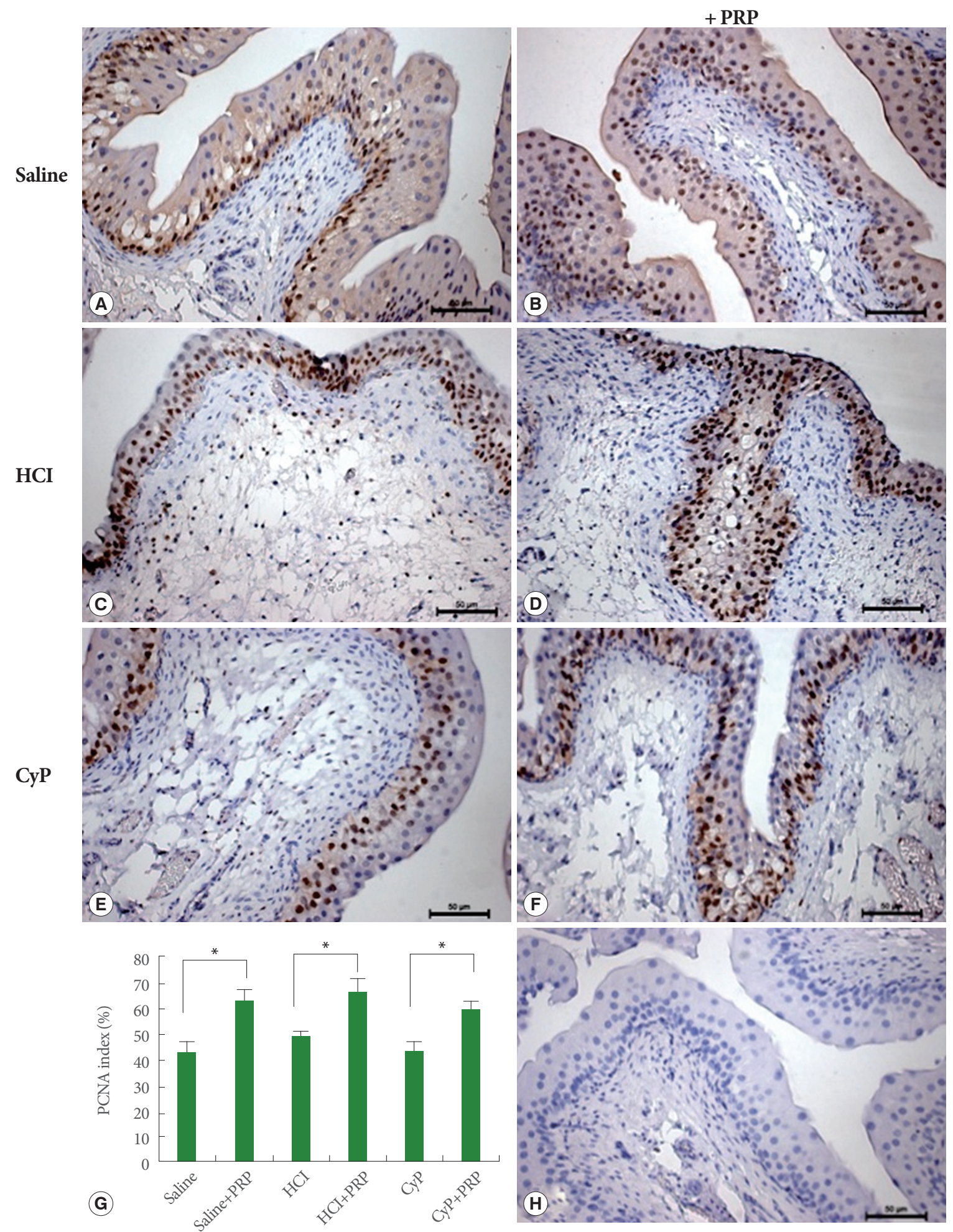

Fig. 4. Proliferating cell nuclear antigen (PCNA) immunoreactivity in the urothelium of the urinary bladder. The number of PCNAimmunopositive cells was lower in the saline (A) and cystitis models (C, E) than in the PRP-administered groups (B, D, F). A significant increase (approximately 20\%) in the PCNA-positive cell number was detected in the urothelium of the PRP-administered groups (G). The bars represent mean \pm standard error of the mean. The negative control where the primary antibody was replaced with its isotype $(\mathrm{H}) .{ }^{*} \mathrm{P}<0.05$. Original magnification, $\times 400$. $\mathrm{PRP}$, platelet-rich plasma; $\mathrm{HCl}$, hydrochloric acid; CyP, cyclophosphamide. 
the etiology, makes the early initiation of treatment of utmost importance. There is no current curative treatment for IC/BPS [9]. The most accepted theory on its pathogenesis is insult to the epithelial glycosaminoglycan layer and loss of integrity of the urothelium, which leads to potassium leakage into the interstitium [10]. This theory gives rise to the hypothesis that the disease must be eradicated while urothelial integrity is restored. The aim of this study was to observe the effect of the intravesical instillation of PRP on damaged urothelium.

In our study, chemical cystitis was successfully achieved using $\mathrm{HCl}$ as described in previous studies, in which dense epithelial loss, hemorrhage, and leukocyte infiltration were observed [11]. No conclusive time lapse has been established between the administration of $\mathrm{HCl}$ and the production of histologic changes; however, 48 hours was assumed to be adequate. Although not supported by microscopic findings, in our study, intravesical instillation of PRP reduced macroscopic hemorrhage. This finding indicates that PRP could be used for hemorrhagic cystitis. The increased mitosis above the basal membrane in the PRP groups indicates a mitotic effect, which is a sign of tissue regeneration. The complete mitotic effect on the urothelium could not be determined because our pilot study was limited to the investigation of the early phases of mucosal regeneration.

With regard to physiological conditions, the urothelium is a very stable type of tissue with a slow proliferative rate [12]. However, after an injury, which causes loss of superficial cells, tight junctions are disrupted, the permeability barrier is interrupted, and the proliferative activity of the remaining basal urothelial cells increases rapidly [13]. The proliferative activity can be evaluated by mitotic figures or available proliferative markers. A proliferation marker, PCNA, is expressed in the nuclei of cells during the DNA synthesis phase of the cell cycle. Increased DNA synthesis followed by mitosis is a hallmark of proliferation. The increased proliferation index assessed by PCNA immunolabeling and the average mitotic index above the basal membrane in the PRP groups indicate a mitotic effect, which is a sign of tissue regeneration. As the proliferative potential decreases, urothelial cell differentiation begins $[14,15]$. The restoration of the urothelial barrier begins with the formation of tight junctions and ends with the terminal differentiation of superficial cells. The achievement of the urothelial integrity throughout the lumen wall could not be presented because our pilot study was limited to the investigation of the early phases of mucosal regeneration. Not only epithelial desquamation but also focal areas with denuded urothelium were observed in our $\mathrm{HCl}$ model.

The use of PRP has been thoroughly investigated, and it is currently being used in many fields of medicine [16-18]. The only clinical study regarding the use of PRP addresses the treatment of vesicovaginal fistula in women; however, that study did not clearly explain the use of PRP [19]. Nevertheless, to the best of our knowledge, this is the first study to use PRP intravesically. The reason underlying the widespread use of PRP is that it consists of PDGF, TGF, IGF, and EGF, which initiate and facilitate wound healing and tissue regeneration. The main goal in the preparation of PRP is to obtain as many thrombocytes as possible in a small amount of plasma. The activation of these thrombocytes leads to the secretion of factors that promote mitogenesis, chemotaxis, differentiation, and metabolism regulation.

This novel pilot study will guide researchers as to how bladder tissue reacts to PRP. The direct contact of PRP with the damaged urothelium, along with the subsequent release of tissue factors and the paracrine effects of these factors, may be considered to initiate cell proliferation at the basal membrane. Previous studies have shown that 10 minutes of PRP contact with damaged mucosa is enough to release $70 \%$ of its mediators [3]. The objective of this study was to establish whether PRP could accelerate the early regeneration of the bladder urothelium. Our findings of increased mitosis and edema in the control group support this argument. Consequently, we hypothesized that PRP exerts its effects via direct contact with the urothelium and passing through the epithelial gap, which initiates local chemotaxis. The elevated mitotic and proliferation indices in undamaged mucosa observed in this study support this hypothesis. Further studies on immunostaining are required to demonstrate the increased expression of the aforementioned healing factors. Moreover, the autologous nature of PRP minimizes possible treatment-related risks.

One of the main limitations of our study was the difficulty in obtaining an adequate volume of unclotted blood from the rabbits. It is crucial to rapidly withdraw blood because the coagulation cascade must not have started at the time of blood sampling. In humans, $20 \mathrm{~mL}$ of blood withdrawal from an antecubital vein can very easily provide 6-8 $\mathrm{mL}$ of PRP. Only $1 \mathrm{~mL}$ of PRP could be generated because only $5 \mathrm{~mL}$ of blood can be obtained from rabbits; however, a marked effect was observed on the bladder urothelium despite this small amount. It is plausible that repeated instillations would have produced even better re- 
sults; naturally, this would require higher numbers of thrombocytes and a larger volume of growth factors.

Another important point that should be considered is the lack of an ideal animal model to mimic IC; the mast cell involvement cannot be imitated in the current animal models. In the future, a better understanding of IC pathophysiology and the mechanism of PRP's effect may be achieved with more appropriate models. Improvements in tissue engineering technologies may allow us to generate better mimicking models.

To conclude, our pilot study showed that PRP increased the mitotic index in the control group and the chemically-induced cystitis model group while decreasing macroscopic bleeding. Our data should be validated by conducting studies that include more subjects, better animal/tissue models, repeated instillations, urodynamic studies, and a standardized instillation volume. The comparison of our findings with those from the literature suggests that the intravesical instillation of PRP is a potential alternative for the treatment of IC and hemorrhagic cystitis.

\section{REFERENCES}

1. Oravisto KJ. Epidemiology of interstitial cystitis. Ann Chir Gynaecol Fenn 1975;64:75-7.

2. Garg AK. The use of platelet-rich plasma to enhance the success of bone grafts around dental implants. Dent Implantol Update 2000; 11:17-21.

3. Marx RE. Platelet-rich plasma: evidence to support its use. J Oral Maxillofac Surg 2004;62:489-96.

4. Sánchez AR, Sheridan PJ, Kupp LI. Is platelet-rich plasma the perfect enhancement factor? A current review. Int J Oral Maxillofac Implants 2003;18:93-103.

5. Matsumoto S, Chichester P, Bratslavsky G, Kogan BA, Levin RM. The functional and structural response to distention of the rabbit whole bladder in vitro. J Urol 2002;168:2677-81.

6. Nagae M, Ikeda T, Mikami Y, Hase H, Ozawa H, Matsuda K, et al. Intervertebral disc regeneration using platelet-rich plasma and biodegradable gelatin hydrogel microspheres. Tissue Eng 2007;13:14758.

7. Juszczak K, Gil K, Wyczolkowski M, Thor PJ. Functional, histological structure and mastocytes alterations in rat urinary bladders fol- lowing acute and [corrected] chronic cyclophosphamide treatment. J Physiol Pharmacol 2010;61:477-82.

8. Fadare O, Yi X, Liang SX, Ma Y, Zheng W. Variations of mitotic index in normal and dysplastic squamous epithelium of the uterine cervix as a function of endometrial maturation. Mod Pathol 2007; 20:1000-8.

9. Fall M, Baranowski AP, Elneil S, Engeler D, Hughes J, Messelink EJ, et al. EAU guidelines on chronic pelvic pain. Eur Urol 2010;57:3548.

10. Parsons CL, Boychuk D, Jones S, Hurst R, Callahan H. Bladder surface glycosaminoglycans: an epithelial permeability barrier. J Urol 1990;143:139-42.

11. Rivas DA, Chancellor MB, Shupp-Byrne S, Shenot PJ, McHugh K, McCue P. Molecular marker for development of interstitial cystitis in rat model: isoactin gene expression. J Urol 1997;157:1937-40.

12. Jost SP. Renewal of normal urothelium in adult mice. Virchows Arch B Cell Pathol Incl Mol Pathol 1986;51:65-70.

13. Veranic P, Erman A, Kerec-Kos M, Bogataj M, Mrhar A, Jezernik K. Rapid differentiation of superficial urothelial cells after chitosaninduced desquamation. Histochem Cell Biol 2009;131:129-39.

14. Romih R, Jezernik K. Endocytosis during postnatal differentiation in superficial cells of the mouse urinary bladder epithelium. Cell Biol Int 1994;18:663-8.

15. Jezernik K, Romih R, Veranic P. Urothelial cell detachment and differentiation in urinary bladder. Pflugers Arch 2000;439(3 Suppl): R135-6.

16. Kajikawa Y, Morihara T, Sakamoto H, Matsuda K, Oshima Y, Yoshida A, et al. Platelet-rich plasma enhances the initial mobilization of circulation-derived cells for tendon healing. J Cell Physiol 2008; 215:837-45.

17. Trink A, Sorbellini E, Bezzola P, Rodella L, Rezzani R, Ramot Y, et al. A randomized, double-blind, placebo- and active-controlled, half-head study to evaluate the effects of platelet-rich plasma on alopecia areata. Br J Dermatol 2013;169:690-4.

18. Carlson NE, Roach RB Jr. Platelet-rich plasma: clinical applications in dentistry. J Am Dent Assoc 2002;133:1383-6.

19. Shirvan MK, Alamdari DH, Ghoreifi A. A novel method for iatrogenic vesicovaginal fistula treatment: autologous platelet rich plasma injection and platelet rich fibrin glue interposition. J Urol 2013;189:2125-9. 